\title{
D2-40 immunohistochemistry in the differential diagnosis of seminoma and embryonal carcinoma: a comparative immunohistochemical study with KIT (CD117) and CD30
}

\author{
Sean K Lau, Lawrence M Weiss and Peiguo G Chu \\ Department of Pathology, City of Hope National Medical Center, Duarte, CA, USA
}

\begin{abstract}
The distinction between seminoma and embryonal carcinoma based on morphology alone can sometimes be problematic, requiring the use of immunohistochemistry to facilitate diagnosis. D2-40 is a monoclonal antibody that reacts with an oncofetal antigen expressed by fetal germ cells and testicular germ cell tumors. The diagnostic value of D2-40 immunohistochemistry in distinguishing seminoma from embryonal carcinoma has not been determined. D2-40 immunoreactivity was evaluated in a series of testicular germ cell tumors and compared with that of KIT (CD117) and CD30, to assess the relative utility of this marker in discriminating between seminoma and embryonal carcinoma. Forty testicular germ cell neoplasms were examined, which included 19 seminomas, three embryonal carcinomas, three teratomas, one yolk sac tumor, and 14 mixed germ cell tumors. The 14 cases of mixed germ cell tumors contained components of seminoma $(n=7)$, embryonal carcinoma $(n=11)$, teratoma $(n=10)$, yolk sac tumor $(n=2)$, and choriocarcinoma $(n=1)$. All cases of pure seminoma and the seminomatous components of mixed germ cell tumors exhibited positive immunoreactivity for D2-40. Focal positivity for D2-40 was also observed in $29 \%$ of the embryonal carcinoma samples. D2-40 immunoreactivity in seminomas was characterized by diffuse membrane staining, whereas for embryonal carcinomas, staining was focal and distributed along the apical surfaces of the neoplastic cells. Immunohistochemical staining for KIT was observed in $92 \%$ of the seminoma samples and in none of the embryonal carcinomas. Conversely, CD30 expression was identified in $93 \%$ of the embryonal carcinoma samples and in none of the seminomas. Other germ cell components showed no immunoreactivity for D2-40, KIT, or CD30. KIT and CD30 are effective immunohistochemical markers in separating seminoma from embryonal carcinoma. Although a highly sensitive marker for seminomas, D2-40 positivity was also observed in a subset of embryonal carcinomas, thus limiting the utility of this antibody for discriminating between these two malignancies.
\end{abstract}

Modern Pathology (2007) 20, 320-325. doi:10.1038/modpathol.3800749; published online 2 February 2007

Keywords: CD30; D2-40; embryonal carcinoma; KIT; seminoma

From a clinical perspective, testicular neoplasms of germ cell origin are generally categorized as either seminomas or nonseminomatous germ cell tumors, based on established differences between these groups with respect to biologic behavior and treatment. In addition to clinical stage, the appropriate therapeutic management of germ cell tumors of the

Correspondence: Dr SK Lau, MD, Department of Pathology, City of Hope National Medical Center, 1500 East Duarte Road, Duarte, CA 91010, USA.

E-mail: SLau@coh.org

Received 20 October 2006; accepted 27 November 2006; published online 2 February 2007 testis is thus largely predicated upon accurate histologic classification. ${ }^{1}$

The histologic diagnosis of seminoma is relatively straightforward in most circumstances due to its characteristic morphologic features. However, some seminomas may exhibit increased nuclear pleomorphism, cell crowding, and lack a lymphocytic infiltrate, resulting in confusion with embryonal carcinoma. $^{2,3}$ Seminoma may also be difficult to distinguish from embryonal carcinoma in the context of a limited biopsy specimen or poor tissue fixation resulting in artifactual morphologic alterations. ${ }^{3}$

In these settings, immunohistochemistry may be utilized to facilitate distinction between seminoma 
and embryonal carcinoma. Evidence suggests that CD30 and KIT (CD117) are useful markers in this regard. CD30 is expressed by the majority of embryonal carcinomas and is generally negative in seminomas, ${ }^{4-7}$ while KIT is regularly expressed in seminomas and only rarely positive in embryonal carcinomas. ${ }^{7,8-11}$ However, CD30 immunoreactivity has been observed in a number of pure seminomas and seminomatous components of mixed germ cell tumors, ${ }^{2,7,12-14}$ and KIT positivity in seminomas has been noted to be variable and weak, ${ }^{15}$ somewhat limiting the diagnostic utility of these particular markers.

D2-40 is a monoclonal antibody that reacts with an oncofetal membrane antigen known as the M2A antigen. ${ }^{16}$ The M2A antigen is present in fetal germ cells of the testis, as well as lymphatic endothelial cells and mesothelial cells. ${ }^{17-19}$ In the context of germ cell neoplasia, the distribution of the M2A antigen has been shown to be largely restricted to intratubular germ cell neoplasia and seminoma, with limited to absent expression in non seminomatous germ cell tumors. ${ }^{16-21}$ The selective expression of the M2A antigen in seminoma suggests potential use of the D2-40 antibody for distinguishing this particular tumor from embryonal carcinoma. In the present study, the immunohistochemical expression of D2-40 was evaluated in testicular germ cell tumors and compared with CD30 and KIT to determine the relative usefulness of this particular marker in discriminating between seminoma and embryonal carcinoma.

\section{Materials and methods}

Forty cases of testicular germ cell tumor were identified from the files of the Department of Pathology, City of Hope National Medical Center, Duarte, CA, USA. Patients ranged in age from 18 to 41 years, with a mean age of 30 years. Hematoxylin and eosin-stained sections from all cases were reviewed to confirm the diagnosis. The testicular germ cell tumors were classified according to the World Health Organization criteria. ${ }^{22}$ The cases included 19 seminomas, three embryonal carcinomas, three teratomas, one yolk sac tumor, and 14 mixed germ cell tumors. The 14 cases of mixed germ cell tumors consisted of the following components: seven seminomas, 11 embryonal carcinomas, 10 teratomas, two yolk sac tumors, and one choriocarcinoma.

A representative tissue block containing tumor was selected from each case for immunohistochemical study. Immunohistochemical staining was performed using the following antibodies: D2-40 (clone D2-40, dilution 1:2, Signet Laboratories, Inc., Dedham, MA, USA), KIT (polyclonal, dilution 1:50, DakoCytomation, Carpinteria, CA, USA), and CD30 (clone Ber-H2, dilution 1:120, DakoCytomation). Sections were deparaffinized in xylene and rehydrated in a graded ethanol series. Antigen retrieval was performed by heating slides in EDTA buffer $(\mathrm{pH}$ 8.0) in a pressure cooker (Biocare Medical, Concord, CA, USA). Staining was performed using an automated immunostainer (DAKO), followed by antibody detection using the DAKO EnVision + System and 3,3'-diaminobenzidine as a chromogen. The slides were counterstained with hematoxylin and coverslipped. Appropriate positive and negative tissue controls were used throughout.

\section{Results}

The immunohistochemical results are summarized in Table 1. All cases of pure seminoma and the seminomatous components of mixed germ cell tumors reacted with the D2-40 antibody. D2-40 immunoreactivity in seminomas was characterized by strong and diffuse membrane staining of the neoplastic cells (Figure 1). Twenty-four of the 26 (92\%) seminoma samples expressed KIT, exhibiting strong membrane, or membrane and cytoplasmic staining patterns. All seminomas were negative for CD30.

Of the 14 samples of embryonal carcinoma studied, 13 were positive for CD30. Three cases of pure embryonal carcinoma were negative for D2-40, while the embryonal carcinoma component of mixed germ cell tumors were positive for D2-40 in four of 11 cases. In contrast to the strong membrane staining observed in seminomas, D2-40 immunoreactivity in the embryonal carcinomas was typically weak, focal, and distributed along the apical or luminal surfaces of the neoplastic cells (Figure 2). All embryonal carcinomas in their pure form and as a component of mixed germ cell tumors were negative for KIT.

Other germ cell tumor components including yolk sac tumor, teratoma, and choriocarcinoma were immunohistochemically negative for D2-40, KIT, and CD30.

Table 1 D2-40, KIT (CD117), and CD30 immunoreactivity in testicular germ cell tumors

\begin{tabular}{lccc}
\hline \multirow{2}{*}{ Tumor type } & \multicolumn{3}{c}{ Number of positive cases } \\
\cline { 2 - 4 } & D2-40 & KIT (CD117) & CD30 \\
& & & \\
\hline Pure germ cell tumor & $19 / 19$ & $18 / 19$ & $0 / 19$ \\
Seminoma & $0 / 3$ & $0 / 3$ & $2 / 3$ \\
Embryonal carcinoma & $0 / 1$ & $0 / 1$ & $0 / 1$ \\
Yolk sac tumor & $0 / 3$ & $0 / 3$ & $0 / 3$ \\
Teratoma & & & \\
& & & \\
Mixed germ cell tumor component & $6 / 7$ & $0 / 7$ \\
Seminoma & $7 / 11$ & $0 / 11$ & $11 / 11$ \\
Embryonal carcinoma & $0 / 2$ & $0 / 2$ & $0 / 2$ \\
Yolk sac tumor & $0 / 10$ & $0 / 10$ & $0 / 10$ \\
Teratoma & $0 / 1$ & $0 / 1$ & $0 / 1$ \\
Choriocarcinoma & & & \\
\end{tabular}



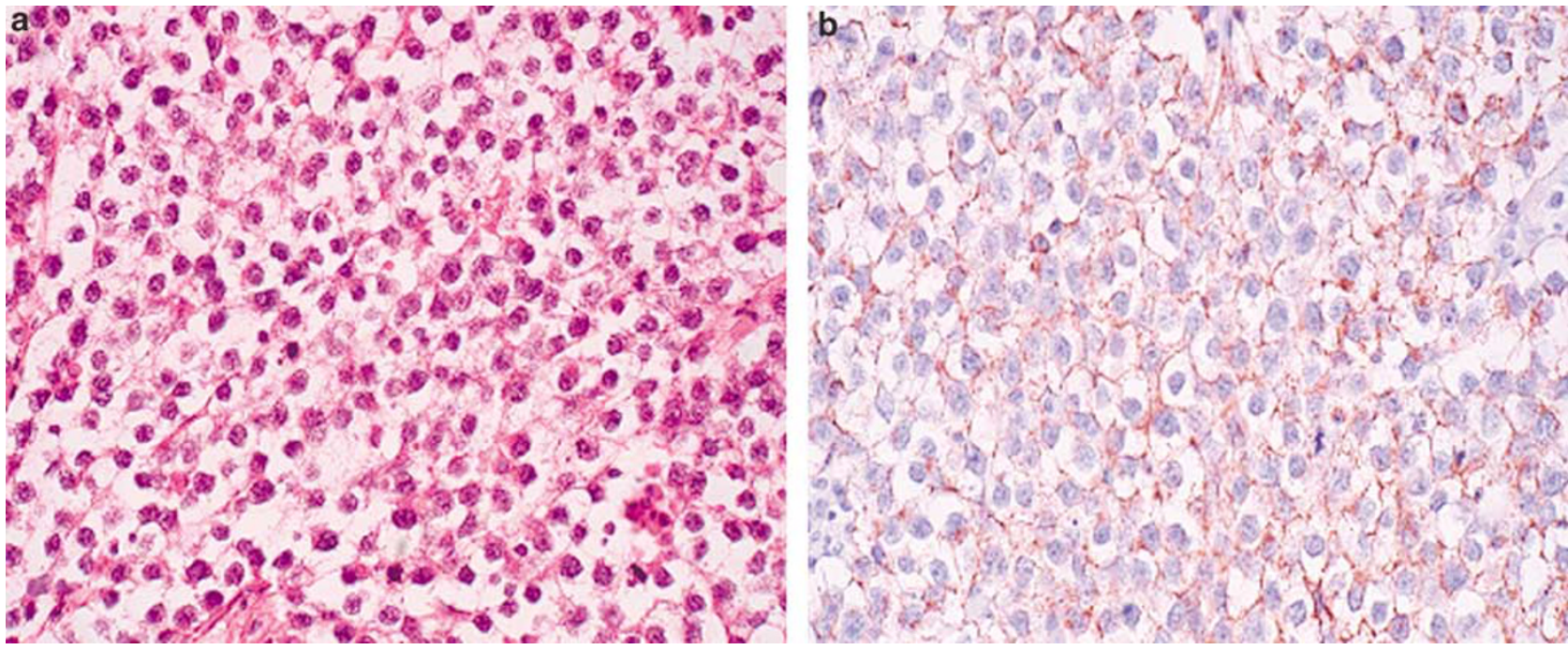

Figure 1 D2-40 immunoreactivity in seminoma. (a) Seminoma, hematoxylin and eosin stain. (b) The seminoma cells exhibit membrane positivity for the D2-40 antibody.
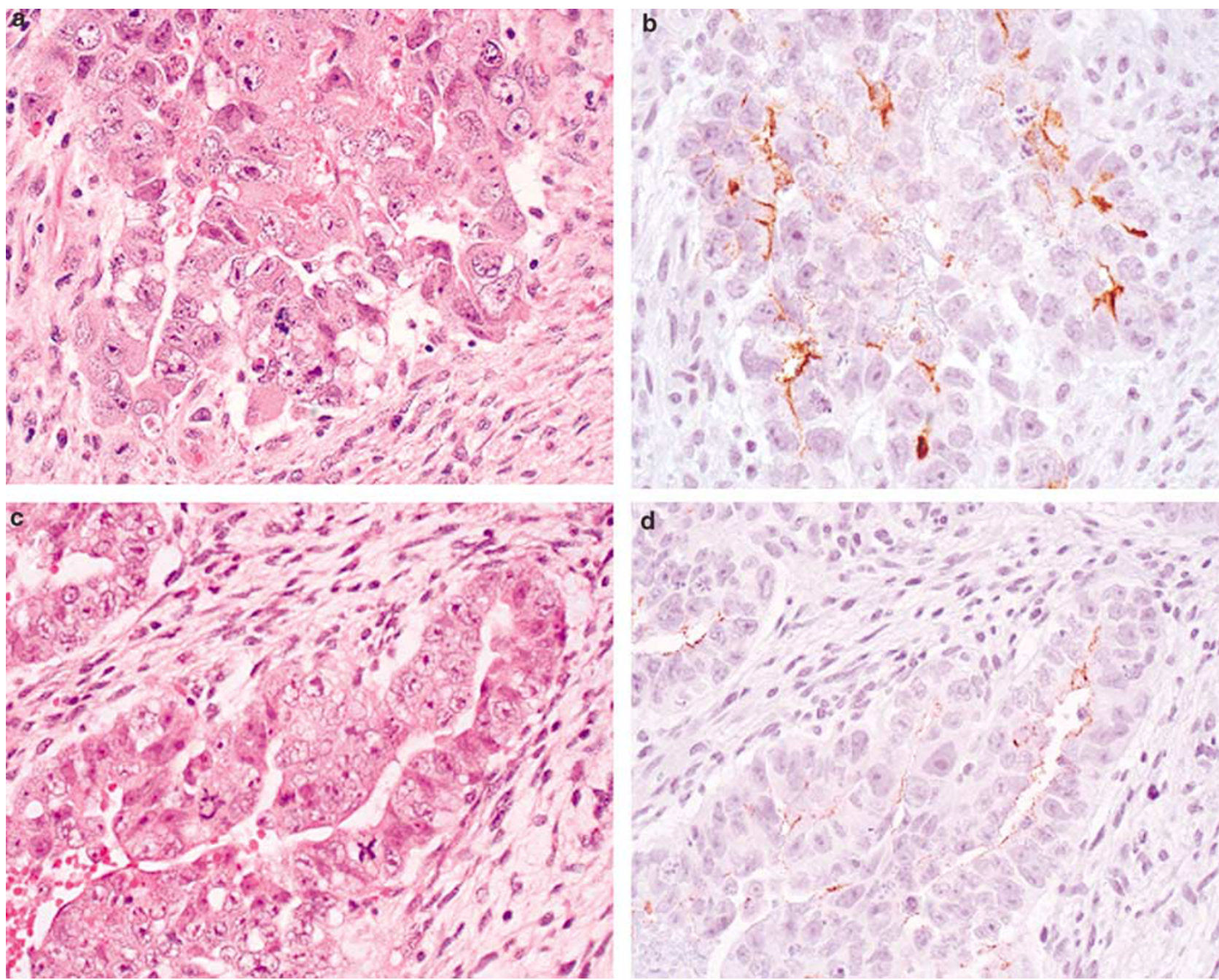

Figure 2 D2-40 immunoreactivity in embryonal carcinoma. (a, c) Embryonal carcinoma, hematoxylin and eosin stain, exhibiting solid (a) and glandular (c) growth patterns. (b, d) Positive staining with the D2-40 antibody is focal in embryonal carcinomas, with immunoreactivity present along the apical/luminal surface of the neoplastic cells. 


\section{Discussion}

D2-40 is a monoclonal antibody which recognizes a $40 \mathrm{kDa} O$-glycosylated sialoglycoprotein with a simple mucin type carbohydrate structure known as the M2A antigen. ${ }^{16}$ Among normal tissues, this antigen has been shown to be present in fetal germ cells, lymphatic endothelium, and mesothelial cells. ${ }^{17-19}$ Initial immunohistochemical investigations performed on frozen tissue specimens using a monoclonal antibody produced against a human ovarian epithelial adenocarcinoma cell line demonstrated M2A antigen expression in all seminomas and seminomatous components of mixed germ cell tumors, but in none of the nonseminomatous germ cell tumors studied. ${ }^{17,18}$

In the present investigation utilizing the D2-40 antibody on paraffin-embedded tissue samples, immunoreactivity was observed in all cases of seminoma, in agreement with the distribution of the M2A antigen established by previous studies using immunohistochemical analysis on frozen sections. ${ }^{17,18}$ However, in contrast to the results of these aforementioned reports indicating an absence of M2A in nonseminomatous germ cell tumors, D240 immunoreactivity was observed in four of 14 (29\%) samples of embryonal carcinoma in the present study. Unlike the diffuse membrane staining present in seminomas, D2-40 immunoreactivity in embryonal carcinomas was always focal and confined to the apical or luminal surfaces of the tumor cells. These findings are comparable to those obtained by other investigators using the D2-40 antibody. ${ }^{15,16}$ Marks et al ${ }^{16}$ reported D2-40 immunoreactivity in $98 \%$ of seminomas, and were also able to demonstrate positivity for this antibody in $69 \%$ of embryonal carcinomas. As in the present study, D240 positivity in embryonal carcinomas was noted by these investigators to be focal and concentrated at the luminal surface of the neoplastic cells, whereas a uniform membrane pattern of staining was observed in seminomas. ${ }^{16}$

Distinguishing seminoma from embryonal carcinoma is important, as differences exist between these two conditions in regards to therapeutic modalities and prognosis. In the majority of cases, distinction between these two entities can be made on a morphologic basis using conventional histologic methods. However, in some situations appropriate histologic classification of testicular germ cell tumors may be problematic. Studies addressing the impact of central histopathologic review of previously diagnosed testicular tumors have demonstrated major discrepancy rates of $4-11 \%$, with the majority of discrepancies attributed to errors in distinguishing seminoma from embryonal carcinoma. ${ }^{23-25}$ The use of immunohistochemistry may therefore be of value in differentiation of these tumor types.

Several immunohistochemical markers with potential utility in differentiating seminoma from embryonal carcinoma have been evaluated previously, among which include antibodies towards various keratins, KIT, and CD30. Early immunohistochemical studies suggested antikeratin antibodies could be useful for the distinction between seminoma and embryonal carcinoma based on observations that, unlike embryonal carcinomas, seminomas lacked keratin intermediate filaments. ${ }^{26-28}$ However, more recent studies utilizing contemporary immunohistochemical techniques on formalin-fixed tissues have indicated keratin expression in seminomas is not unusual, with keratin positivity ranging from 4 to $45 \%$ depending on the particular antibody employed., ${ }^{2,5,13,14}$ Thus, keratin expression alone cannot be used to definitively distinguish seminoma from embryonal carcinoma. More effective immunohistochemical markers in this regard appear to be KIT and CD30.

In the context of testicular germ cell neoplasia, KIT immunoreactivity has been shown to be largely limited to seminomas, with positivity in non seminomatous germ cell tumors considered uncommon, and characterized by focal cytoplasmic, rather than the typical membrane pattern of staining. ${ }^{7-11}$ Previous immunohistochemical studies have demonstrated KIT expression in up to $100 \%$ of cases of seminoma. ${ }^{9-11}$ In the present study, $92 \%$ of seminomas were positive for KIT, while none of the embryonal carcinomas or other histologic types of non seminomatous germ cell tumors were noted to express this marker, in keeping with the findings of previous investigators. Although one study has observed heterogeneous and weak staining of seminomas using the KIT antibody, ${ }^{15}$ in our experience, KIT reactivity in seminomas was typically strong and diffuse.

CD30 is regarded as a sensitive as well as a specific maker for embryonal carcinoma, with other types of germ cell tumors, including seminoma, generally lacking CD30 expression..$^{4-7}$ Similar to previous reports, CD30 immunoreactivity was restricted to only pure embryonal carcinomas and the embryonal carcinoma components of mixed germ cell tumors in the current study.

Although not observed in the present investigation, it should be recognized that focal CD30 expression has been described in a subset of seminomas, ${ }^{2,7,12-14}$ thus somewhat limiting the specificity of CD30 as an individual marker for embryonal carcinoma. As such, an antibody panel consisting of CD30 in combination with KIT has been suggested by Leroy et $a l^{7}{ }^{7}$ to account for any shortcomings in the use of these particular markers individually in the distinction of seminoma from embryonal carcinoma. In that study, a KIT positive, CD30 negative immunophenotype was characteristic of seminoma, while a KIT negative, CD30 positive immunophenotype was indicative of embryonal carcinoma. In addition, none of the seminomas expressed CD30 in the absence of KIT, and none of the embryonal carcinomas expressed KIT in 
the absence of CD30. The results of the present study confirm these previous observations regarding the differential expression of these particular markers in seminoma and embryonal carcinoma. In all, $92 \%$ of the seminoma samples were positive for KIT and negative for CD30, while conversely $93 \%$ of the embryonal carcinoma samples were positive for CD30 and negative for KIT.

The data obtained in the current investigation suggest D2-40 may serve as a useful immunohistochemical marker for the identification of seminoma. D2-40 was commonly expressed in seminomas, with an observed sensitivity higher than that of KIT in the present study. However, D240 immunoreactivity was also observed in a subset of embryonal carcinomas, making this a less specific marker for seminoma than KIT. It is nonetheless important to note that the pattern of D2-40 positivity in seminomas was distinct from that observed in embryonal carcinomas. Reactivity for D2-40 in seminomas was typically diffuse and membranous, while in embryonal carcinomas, positivity was always focal and limited to the apical or luminal surface of the cells. However, despite these differences, the fact that nearly one-third of the embryonal carcinomas were D2-40 positive in the present investigation limits the diagnostic utility of this marker in differentiating embryonal carcinoma and seminoma.

In summary, antibodies to KIT and CD30 constitute a useful panel of markers that allows for seminoma to be distinguished from embryonal carcinoma. KIT expression in the absence of CD30 immunoreactivity would substantiate a diagnosis of seminoma, while conversely, CD30 positivity with lack of KIT expression would be consistent with embryonal carcinoma. Although a highly sensitive marker of seminomas, focal D2-40 immunoreactivity can be seen in a subset of embryonal carcinomas, thus limiting the practical value of this marker for discriminating between these particular malignancies.

\section{References}

1 Parkinson MC, Harland SJ, Harnden P, et al. The role of the histopathologist in the management of testicular germ cell tumour in adults. Histopathology 2001;38: 183-194.

2 Tickoo SK, Hutchinson B, Bacik J, et al. Testicular seminoma: a clinicopathologic and immunohistochemical study of 105 cases with special reference to seminomas with atypical features. Int J Surg Pathol 2002;10:23-32.

3 Ulbright TM. Germ cell tumors of the gonads: a selective review emphasizing problems in differential diagnosis, newly appreciated, and controversial issues. Mod Pathol 2005;18:S61-S79.

4 Pallesen G, Hamilton-Dutoit SJ. Ki-1 (CD30) antigen is regularly expressed by tumor cells of embryonal carcinoma. Am J Pathol 1988;133:446-450.
5 Ferreiro JA. Ber-H2 expression in testicular germ cell tumors. Hum Pathol 1994;25:522-524.

6 Durkop H, Foss HD, Eitelbach F, et al. Expression of the CD30 antigen in non-lymphoid tissues and cells. J Pathol 2000;190:613-618.

7 Leroy X, Augusto D, Leteurtre E, et al. CD30 and CD117 (c-kit) used in combination are useful for distinguishing embryonal carcinoma from seminoma. J Histochem Cytochem 2002;50:283-285.

8 Tsuura Y, Hiraki H, Watanabe K, et al. Preferential localization of c-kit product in tissue mast cells, basal cells of skin, epithelial cells of breast, small cell lung carcinoma and seminoma/dysgerminoma in human: immunohistochemical study on formalin-fixed, paraffinembedded tissues. Virchows Arch 1994;424:135-141.

9 Izquierdo MA, Van der Valk P, Van Ark-Otte J, et al. Differential expression of the c-kit proto-oncogene in germ cell tumours. J Pathol 1995;177:253-258.

10 Strohmeyer T, Reese D, Press M, et al. Expression of the c-kit proto-oncogene and its ligand stem cell factor (SCF) in normal and malignant human testicular tissue. J Urol 1995;153:511-515.

11 Tian Q, Frierson Jr HF, Krystal GW, et al. Activating c-kit gene mutations in human germ cell tumors. Am J Pathol 1999;154:1643-1647.

12 Hittmair A, Rogatsch H, Hobisch A, et al. CD30 expression in seminoma. Hum Pathol 1996;27:1166-1171.

13 Suster S, Moran CA, Dominguez-Malagon $\mathrm{H}$, et al. Germ cell tumors of the mediastinum and testis: a comparative immunohistochemical study of 120 cases. Hum Pathol 1998;29:737-742.

14 Cheville JC, Rao S, Iczkowski KA, et al. Cytokeratin expression in seminoma of the human testis. Am J Clin Pathol 2000;113:583-588.

15 Biermann K, Klingmuller D, Koch A, et al. Diagnostic value of markers M2A, OCT3/4, AP-2gamma, PLAP and c-KIT in the detection of extragonadal seminomas. Histopathology 2006;49:290-297.

16 Marks A, Sutherland DR, Bailey D, et al. Characterization and distribution of an oncofetal antigen (M2A antigen) expressed on testicular germ cell tumours. Br J Cancer 1999;80:569-578.

17 Bailey D, Baumal R, Law J, et al. Production of a monoclonal antibody specific for seminomas and dysgerminomas. Proc Natl Acad Sci USA 1986;83: 5291-5295.

18 Bailey D, Marks A, Stratis M, et al. Immunohistochemical staining of germ cell tumors and intratubular malignant germ cells of the testis using antibody to placental alkaline phosphatase and a monoclonal antiseminoma antibody. Mod Pathol 1991;4:167-171.

19 Sonne SB, Herlihy AS, Hoei-Hansen CE, et al. Identity of M2A (D2-40) antigen and gp36 (Aggrus, T1A-2, podoplanin) in human developing testis, testicular carcinoma in situ and germ-cell tumours. Virchows Arch 2006;449:200-206.

20 Giwercman A, Marks A, Bailey D, et al. A monoclonal antibody as a marker for carcinoma in situ germ cells of the human adult testis. APMIS 1988;96:667-670.

21 Giwercman A, Cantell L, Marks A. Placental-like alkaline phosphatase as a marker of carcinoma-in-situ of the testis. Comparison with monoclonal antibodies M2A and 43-9F. APMIS 1991;99:586-594.

22 Eble JN, Sauter G, Epstein JI, et al. World health organization classification of tumours. Pathology and Genetics of Tumours of the Urinary System and Male Genital Organs. IARC Press: Lyon, 2004. 
23 Segelov E, Cox KM, Raghavan D, et al. The impact of histological review on clinical management of testicular cancer. Br J Urol 1993;71:736-738.

24 Lee AH, Mead GM, Theaker JM. The value of central histopathological review of testicular tumours before treatment. BJU Int 1999;84:75-78.

25 Delaney RJ, Sayers CD, Walker MA, et al. The continued value of central histopathological review of testicular tumours. Histopathology 2005;47: 166-169.

26 Battifora H, Sheibani K, Tubbs RR, et al. Antikeratin antibodies in tumor diagnosis. Distinction between seminoma and embryonal carcinoma. Cancer 1984; 54:843-848.

27 Ramaekers F, Feitz W, Moesker O, et al. Antibodies to cytokeratin and vimentin in testicular tumour diagnosis. Virchows Arch A Pathol Anat Histopathol 1985; 408:127-142.

28 Nakagawa Y, Perentes E, Ross GW, et al. Immunohistochemical differences between intracranial germinomas and their gonadal equivalents. An immunoperoxidase study of germ cell tumors with epithelial membrane antigen, cytokeratin, and vimentin. J Pathol 1988;156:67-72. 